\title{
042
}

\section{APPROFONDIMENTI DIAGNOSTICI ED EZIOPATOGENETICI SU BATTERI "CORINEFORMI" IN CORSO DI BATTERIEMIE E/O SEPSI}

\author{
Dell'Anna P., Zerbini L., Rossi S., Buttrini M., Somenzi P., \\ Menozzi M.G., Chezzi C. e Dettori G.
}

\author{
Dipartimento di Patologia e Medicina di Laboratorio, \\ Sezione di Microbiologia, Università degli Studi di Parma, \\ Viale A. Gramsci 14, 43100 Parma
}

\section{Introduzione.}

Studi recenti hanno evidenziato un'aumentata incidenza delle batteriemie ed uno spostamento nella loro eziologia. Obiettivo del presente lavoro è stato quello di approfondire gli aspetti microbiologici nonché clinico-epidemiologici di specie di raro riscontro e, talvolta, di difficile inquadramento tassonomico, quale quelle dei "corineformi", connessi con le batteriemie nell'uomo.

\section{Metodi.}

Sono stati analizzati (gennaio 2003 - luglio 2004) 12.147 campioni di sangue provenienti da 4.427 pazienti secondo il protocollo diagnostico in uso. E' stato rivisto il percorso analitico, che ha condotto alla definitiva identificazione di specie di "corineformi" isolati dal sangue, seguendo le recenti indicazioni di Funke G. et al. (2003).

\section{Risultati.}

Sono risultati positivi 1.989 campioni $(16,4 \%)$, provenienti da 1.175 pazienti, con almeno un'indagine emocolturale positiva (26,5\% dei pazienti esaminati). Nell'ambito dei batteri Gram-positivi (72\% dei ceppi isolati) sono stati riscontrati 24 ceppi di batteri "corineformi", provenienti da altrettanti pazienti, che hanno dimostrato di appartenere a 8 differenti specie. Risultati concordanti sono stati ottenuti nell'identificazione di Corynebacterium accolens, C. propinquum, C. urealyticum e C. striatum/amycolatum mediante API Coryne (bioMérieux, Francia) nonchè applicando lo schema identificativo pubblicato da Funke, che prevede la valutazione del profilo biochimico e di altre caratteristiche morfologiche e/o metabolico-enzimatiche. Al contrario, sono stati identificati Arcanobacterium pyogenes, C. falsenii e C. simulans solo attraverso quanto indicato dall'Autore sopra citato. L'analisi accurata degli aspetti clinico-microbiologici, condotta sui 24 casi osservati ha rivelato tre casi di vera batteriemia (Arcanobacterium pyogenes, C. striatum e C. urealyticum) e quattro pseudobatteriemie.

Conclusioni.

Come è stato osservato in questo studio per i batteri "corineformi", i sistemi identificativi attualmente a disposizione da soli possono risultare non conclusivi quando i generi e/o i gruppi di specie esaminati hanno subito importanti cambiamenti tassonomici attraverso l'applicazione di metodologie molecolari in laboratori di ricerca di riferimento. Elevata $(66,7 \%$ dei casi) è stata la percentuale dei ceppi risultati contaminanti. 